\title{
Die Risikogesellschaft
}

- Ein vernachlässigtes

Konzept in der europäischen

\section{Stagnationsdiskussion}




\title{
Die Risikogesellschaft - Ein vernachlässigtes Konzept in der europäischen Stagnationsdiskussion
}

\author{
Gunther Tichy
}

\section{Keywords}

Risikogesellschaft, Wirtschaftspolitik, Vergleich USA/EU/Skandinavien, Deregulierung, Vertrauen, risk society, economic policy, comparison USA/EU/Scandinavia, dergulation, trust

\begin{abstract}
Europa befindet sich - wie schon in den späten sechziger oder den frühen achtziger Jahren - abermals in einer Stagnationsphase und fällt in seiner Wirtschaftsleistung hinter die USA zurück. Das Phänomen wird auf beiden Seiten des Atlantik hitzig diskutiert und zumeist auf ein Nachhinken Europas bei Deregulierung und Privatisierung, auf überhöhte Sozialausgaben und einen Rückstand bei neuen Technologien zurückgeführt, zuweilen auch auf restriktivere Konjunkturpolitik. Alle diese Erklärungen sind nicht unrichtig, können aber aus zwei Gründen nicht genügen: Erstens vernachlässigen sie die erheblichen Unterschiede innerhalb Europas: Wachstumsschwach sind vor allem die drei großen EU-Staaten, wogegen vor allem die skandinavischen Staaten keineswegs langsamer wachsen als die USA. Zweitens greifen die Argumente insofern zu wenig tief, als sie auf die Ursachen der behaupteten Mängel nicht eingehen. Die vorliegende Arbeit versucht zu zeigen, dass hinter allen drei Erklärungsversuchen der europäischen Stagnation - Reformscheu, Innovationsscheu und inflationsfixierte Konjunkturpolitik - eine wichtige Ursache in der tiefliegenden Verunsicherung der Bevölkerung und der daraus resultierenden Risikoscheu liegt. Umfragen lassen klar erkennen, dass die Verunsicherung in den wachstumsschwachen großen Ländern Deutschland, Frankreich und Italien merklich stärker ausgeprägt ist als etwa in Skandinavien. Selbst wenn die Kausalität von der Wachstumsschwäche zur Verunsicherung laufen sollte, wäre das für die Wirtschaftspolitik nicht irrelevant, desto mehr, wenn - wie zu zeigen versucht wird - Attentismus und Stagnation Folgen einer, nicht zuletzt (wirtschafts-)politisch ausgelösten Verunsicherung sind.
\end{abstract}

(c) ITA - Institut für Technikfolgen-Abschätzung der Österreichischen Akademie der Wissenschaften A- 1030 Wien, Strohgasse 45/5, Fax: +43-1-515 8 I http://www.oeaw.ac.at/ita/ 


\section{Inhalt}

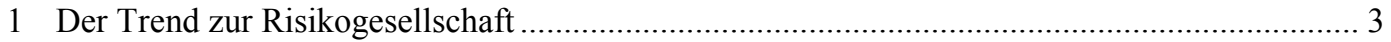

2 Die Entwicklung des sozialwissenschaftlichen Risikobegriffs ............................................ 4

3 Die sinkende Risikoakzeptanz der Öffentlichkeit............................................................. 7

4 Sozialwissenschaftliche Ansätze zur Erklärung der zunehmenden Risikoaversion.................. 10

5 Zunehmende Verunsicherung als Ursache der europäischen Stagnation................................ 11

6 Mangelndes Systemvertrauen in den großen EU-Staaten ............................................... 15

7 Die Vernachlässigung der ,Risiko'-Diskussion durch die Volkswirte..................................... 18

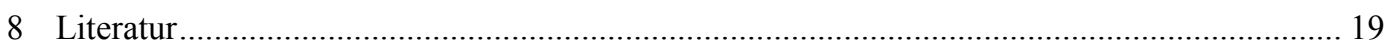

Der Autor dankt Georg Aichholzer, Karl Aiginger und Helge Torgersen für Kritik und wertvolle Anregungen. 


\section{Der Trend zur Risikogesellschaft}

Den Sozialwissenschaften ist seit langem bewusst, dass das Bedürfnis nach Sicherheit im Lauf der letzten drei Jahrzehnte weltweit zugenommen hat; das Schlagwort von der Risikogesellschaft (Beck 1986) wurde von den Massenmedien übernommen, und ist Bestandteil der Alltagssprache geworden. In Europa ist dieses Sicherheitsbedürfnis besonders stark ausgeprägt, doch mit markanten regionalen Unterschieden: Manche Staaten, etwa die skandinavischen, konnten die Folgen besser abfangen als die großen Flächenstaaten, in denen die Verunsicherung ein bedenkliches Ausmaß erreicht hat. Volkswirtschaftslehre und Wirtschaftspolitik vernachlässigen diese Entwicklung jedoch sträflich. Der Mainstream ignoriert selbst die unsicherheitstheoretischen Ansätze, die Volkswirte in den zwanziger und dreißiger Jahren des vorigen Jahrhunderts entwickelten, und konzentriert sich auf Rationale Erwartungen, einen Ansatz, der für das strukturkonstante Wachstum der sechziger Jahre passte, nicht jedoch auf die „Neue Unübersichtlichkeit“ (Habermas 1985) unserer Tage. Um die Folgen des unbefriedigten Bedürfnisses nach Sicherheit auf die europäische Stagnation aufzuzeigen, muss diese Arbeit etwas weiter ausholen. Sie muss zunächst die sozialwissenschaftliche Risikodebatte nachzeichnen (Abschnitt 2), in der der wissenschaftlich unbrauchbare Begriff , Sicherheit' durch die Begriffe ,Gefahr' und ,Risiko' ersetzt, und beide als soziale Konstrukte erkannt, somit endogenisiert wurden. Die üblichen Erklärungen des Trends zur Risikogesellschaft (Abschnitt 3) müssen auf die in den zentralen sozialwissenschaftlichen Ansätzen vorgeschlagenen tieferen Ursachen zurückgeführt werden (Abschnitt 4). Erst auf dieser Basis kann die Stagnation der großen europäischen Staaten aus zunehmender Verunsicherung infolge erheblich höherer Sicherheitsbedürfnisse erklärt werden (Abschnitt 5), die günstige Entwicklung der skandinavischen Staaten umgekehrt aus geringerer Verunsicherung als Folge ihres größeren Systemvertrauens (Abschnitt 6).

Empirisch ist die Entwicklung Europas zur Risikogesellschaft - obwohl offensichtlich - nicht leicht zu fassen. Analytisch lässt sich dieser Trend bei einem Vergleich der Diskussionen der sechziger Jahre des vorigen Jahrhunderts mit denjenigen des neuen Jahrtausends erkennen: Damals glaubte man an die Steuerbarkeit der Wirtschaft, an Wirtschaftswachstum, technischen Fortschritt und sozialen Ausgleich; man war überzeugt, dass es den Kindern besser gehen würde, ein Optimismus der heute völlig verschwunden ist; die „Neue Unübersichtlichkeit“ und der Generationenkonflikt sind an seine Stelle getreten (Tichy 2004). Quantitativ-statistisch ist das - trotz erheblicher Zunahme von Einkommen, Lebensstandard und Lebensqualität ${ }^{1}$ - ausgeprägte und weiter zunehmende Sicherheitsbedürfnis allerdings schwer zu fassen. Ein Indikator ist das abnehmende Vertrauen, das Himmelfarb (1995) für Großbritannien nachweisen konnte. Ein weiterer europäischer Indikator ist die hohe und bis vor kurzem weiter steigende Sozialquote, ${ }^{2}$ und vor allem der heftige Widerstand gegen deren Senkung - trotz der vielbeklagt hohen Beiträge ist für $60 \%$ der Österreicher soziale Sicherheit sehr wichtig (GfK 2002); ein weiterer sind die, trotz der höheren sozialen Absicherung, geradezu explodierenden Ausgaben für Privatversicherungen. ${ }^{3}$ Umfragen zeigen ein steigendes Bedürfnis nach physischer Sicherheit, ${ }^{4}$ konservativ-absichernde Werte haben Konjunktur. ${ }^{5}$

1 Der Wohlstand steigt trotz zeitweise unbefriedigendem Wachstum: Die Realeinkommen/Kopf erreichten 2000 in Österreich bzw. Deutschland das 11/2- bzw. 11/4-fache des Jahres 1965; die Freizeit der Österreicher stieg zwischen 1979 und 2002 von 3 auf 43/4 Stunden/Tag (IMAS 2002b), die Lebenserwartung bei Geburt zwischen 1965 und 2000 von 66 1/2 auf 751/2 (Männer) bzw. von 73 auf 81 Jahre (Frauen).

2 In der EU zwischen 1990 und 2000 von 25,5 \% auf 27,3 \%, in Österreich in dem längeren Zeitraum 1965 bis 2000 von 19,8 \% auf 28,2 \%; erst in den letzten Jahren beginnt sie in einigen Ländern zu sinken.

3 Die Bruttoprämien ohne Autoversicherung stiegen in Österreich zwischen 1965 und 2001 auf das rund dreißigfache (die Preise haben sich bloß vervierfacht).

4 Deutsche Befragungen zeigen, dass der Schutz vor Kriminalität in der Zielhierarchie sehr weit oben rangiert, und die Menschen mit der öffentlichen Sicherheit wenig zufrieden sind (Huschka 2002, 18). 
Der Trend zunehmender Verunsicherung ist keineswegs auf Europa beschränkt: Risiken werden überall intensiver wahrgenommen (Slovic [1997] 2000d, 390). Auch in den USA hat das Vertrauen abgenommen (Putnam 2000), ${ }^{6}$ die Bevölkerung sieht sich größeren Risken ausgesetzt als in der Vergangenheit, und erwartet noch größere in der Zukunft (Harris 1980). Anders als in den meisten europäischen Staaten konnten die Folgen des Verunsicherungs-Trends in den USA jedoch durch expansive Konjunkturpolitik und höhere Investitionen in moderne Technologien aufgefangen werden.

\section{Die Entwicklung des sozialwissenschaftlichen Risikobegriffs}

Dass sich das steigende Sicherheitsbedürfnis der Risikogesellschaft nicht quantifizieren und bloß vage beschreiben lässt, hängt mit der mangelnden Präzision des Begriffs zusammen. Wie Kaufmann $(1970,176)$ wohl als einer der Ersten herausarbeitete, ist Sicherheit ,ein vorwissenschaftlicher Begriff, ohne analytische Potenz" (ebd. 298): In der amerikanischen Diskussion tauche er in den dreiBiger Jahren auf, in Deutschland würde er seit den fünfziger Jahren gehäuft verwendet (ebd. 7). Anders als im Englischen, das zwischen security (Sicherheit), safety (technische Sicherung) und certainty (Gewissheit) differenziert, würde der Begriff im Deutschen undifferenziert-umfassend verwendet (ebd. 90), eher normativ verstanden, und habe ,emotionale Appellqualität“ (ebd. 36), ,[d]as Wort ,Sicherheit' ist das Symbol einer gesellschaftlichen Wertidee geworden“ (ebd. 10); ${ }^{7}$ als solche sei sie wissenschaftlich aber nicht operationalisierbar und als Zielgröße ungeeignet. Es ,darf die These gewagt werden, dass für das moderne Bewusstsein Sicherheit überhaupt nur als Nicht-Unsicherheit positiv bestimmt werden kann“" (ebd. 175, meine Hervorhebung G.T.). ${ }^{8}$

Infolge der Mängel des Begriffs ,Sicherheit' konzentrierte sich die sozialwissenschaftliche Diskussion auf das Konzept ,Risiko', ein Begriff der zunehmend häufig, ${ }^{9}$ allerdings recht unterschiedlich verwendet wird. Die volkswirtschaftliche Literatur unterscheidet seit Knight (1921) zwischen Risiko und Unsicherheit: Risiko bezeichnet wiederholte Abfolgen bestimmter Situationskombinationen, deren Wahrscheinlichkeit abgeschätzt werden kann, Unsicherheit hingegen die Konfrontation mit weitgehend neuen oder einmaligen Konstellationen, bei denen das Wahrscheinlichkeitskalkül versagt. Die soziologische Literatur interessiert sich weniger für Eintrittswahrscheinlichkeiten: Luh-

5 Begriffe wie „Sicherheit“, „Heimat“, „Ordnung“ und „Stabilität“" sind rund $80 \%$ der Österreicher sympathisch, ,dynamische' Begriffe wie „Wettbewerb“ und „Leistungsstreben“ hingegen bloß knapp über $50 \%$ (IMAS 2002a); die mit vermuteten Bedrohungen verbundenen Begriffe „Genforschung“ (21\%), ,Islamismus“ (20\%) und „Kernenergie“ (14\%) sind die am wenigsten sympathischen (IMAS 2002a); $54 \%$ der Österreicher ist der technische Fortschritt zu rasch (IMAS 2001).

6 Genauer: die Abnahme des „social capital“, das - je nach Indikator - 1997 um $20 \%$ bis $50 \%$ niedriger war als 1960 .

7 Kaiser Wilhelm I an den Reichstag: „dem Vaterlande neue und dauernde Bürgschaften seines inneren Friedens und den Hilfsbedürftigen größere Sicherheit und Ergiebigkeit des Beistandes ... zu hinterlassen“- soziale Sicherung wurde somit bereits als Voraussetzung der gesellschaftlichen Integration verstanden!

8 Im gesellschaftlichen Sprachgebrauch findet Kaufmann (1970, 341) drei Konzepte von Sicherheit: ,,(1) Retrospektiv als ,Sicherheit und Geborgenheit', (2) paradigmatisch als Systemsicherheit, berechenbare Verfügbarkeit von Mitteln zu beliebigen Zwecken, und (3) psychologisch als Selbstsicherheit, Leitbild subjektiver Identität. Gemeinsam ist ihnen das Konzept einer „Zukunft, in der nicht ,alles möglich’ ist“.

9 Im Social Citation Index stieg die Zahl der Nennungen kontinuierlich auf 6000 im Jahre 1982 (Inhaber and Norman 1982) und es gibt eigene, diesem Thema gewidmete Zeitschriften. 
mann (1993, 327f) hält die Zurechnung für relevant, und unterscheidet insofern zwischen Gefahr und Risiko: Bei beiden ,handelt es sich um etwaige künftige Schäden, deren Eintritt gegenwärtig unsicher und mehr oder weniger unwahrscheinlich ist. Bei Gefahren wird der Schadenseintritt der Umwelt zugerechnet, bei Risiken wird er als Folge eigenen Handelns oder Unterlassens gesehen“ (Luhmann [1988] ${ }^{2}$ 1996, 269). Gefahren resultieren nicht bloß aus Naturkatastrophen, sondern können auch durch Entscheidungen Anderer ,sozial erzeugt' werden: Gentechnik ist für den GenWissenschafter - aus der Entscheiderperspektive - Risiko, für die Bevölkerung - aus der Betroffenheitsperspektive - Gefahr; daraus ergibt sich zwangsläufig ein struktureller Konflikt zwischen Experten- und Laienmeinung, ein double standard zwischen , freiwillig eingegangen' und , aufgezwungen', eine der zentralen Ursachen der zunehmenden Verunsicherung die von der Knight'schen Unterscheidung nicht erfasst wird. Die sozialwissenschaftliche Literatur, die sich mit den Gefahren der modernen Technik bzw. Wirtschaft für Umwelt und Natur beschäftigt, folgt weitgehend der Luhmann'schen Terminologie und spricht generell von Risiken, auch dort, wo Eintrittswahrscheinlichkeiten - vor allem mangels genügender Information - sinnvoll gar nicht abgeschätzt werden können, ein - wie sich zeigen wird - entscheidender Aspekt.

In ihrer heutigen Definition sind Gefahr und Risiko relativ moderne Begriffe. ${ }^{10}$ Die auf der von B. Pascal und P. de Fermat entwickelten Wahrscheinlichkeitsrechnung beruhende Abschätzung des technischen Risikos ist seit dem 17. Jahrhundert bekannt (Wiedemann 1993, 56), etwa in Form der Wahrscheinlichkeit von Sterbefällen (Sterbetafeln); sie war konstitutiv für das Entstehen von Versicherungen. Geschätzt wird dieses , objektive' Risiko, damals wie heute, als Schadenshöhe mal Eintrittswahrscheinlichkeit. Für normale Versicherungsrisiken ${ }^{11}$ genügt das; bei der Übertragung dieses Konzepts auf das ,Risiko’ von Krisen - und damit auf die Phänomene Unsicherheit bzw. Gefahr - traten jedoch konzeptuelle Probleme auf. Sie betreffen vor allem die Abschätzung der Eintrittswahrscheinlichkeit (ungenügende Zahl von Fällen), die Abschätzung von Systemrisiken komplexer Anlagen (etwa Atomkraftwerke), die Monetarisierung der Schadenshöhe, ${ }^{12}$ das Fehlen einer identischen Messskala für Schaden und Nutzen, sowie die außerhalb der Fachwelt nicht akzeptierte Implikation, dass hoher Schaden mit kleiner Eintrittswahrscheinlichkeit gleiches ,Risiko' bedeute wie kleiner Schaden mit hoher Eintrittswahrscheinlichkeit (Wynne 1987, 274ff; Binswanger 1990).

Unbeschadet all dieser Probleme und der mangelnden Akzeptanz der ,objektiven' Risikodefinition durch die Öffentlichkeit wird sie von 'risk professionals' - i.d.R. Naturwissenschaftern oder Technikern - zur Abschätzung von Gefahren etwa im Bereich Umwelt oder Atomanlagen verwendet (Dietz and Rycroft 1987). Die Ergebnisse rechtfertigen die Skepsis der Öffentlichkeit: Jungermann $(1991,349)$ etwa fand in allen fünf von ihm untersuchten Bereichen ${ }^{13}$ unterschiedliche Konzeptualisierungen von ,Risiko' durch Experten in Bezug auf Art, Definition, Operationalisierung und Gewichtung mehrdimensionaler Schäden sowie unterschiedliche, vielfach nicht-quantitative Definitionen von Unsicherheit. Ein Experiment der Gemeinsamen Forschungsstelle der EG im Jahre 1986 zeigte die daraus resultierende enorme Varianz der Expertenschätzungen (Otway and v. Winterfeld

10 Früher verstand man darunter Anderes: ,'Die Sicheren' bei Luther waren die, die nicht auf Gott vertrauen, die sich fälschlicherweise geschützt fühlen. Seit dem 19. Jahrhundert ist dieser Wertbegriff verschwunden, Sicherheit somit aus der moralischen Ambivalenz entlassen, die darauf beruhte, dass Gott und nicht der Mensch der eigentlich Handelnde ist“ (Kaufmann 1970, 64ff). Demgemäß fiel „[d]ie Geburtsstunde des Begriffs des Risikos ... zusammen mit einer historischen Epoche, in der die Welt erstmals als eine durch menschliches Handeln veränderbare begriffen wurde, in der Strukturen durch Handeln bewusst zur Disposition gestellt werden konnten ... um einer Chance willen“" (Evers und Nowotny 1987).

11 Bei denen also die Eintrittswahrscheinlichkeit bekannt ist.

12 Etwa die Abschätzung der ,Kosten' eines Menschenlebens oder der Klimaerwärmung.

13 Gentechnologie, Kernenergie, Chemie, Informations- und Kommunikationstechnologien sowie anthropogen induzierte Klimaveränderungen. 
1992). ${ }^{14}$ Divergierende Experteneinschätzungen resultieren weiters aus der Faszination der meisten Wissenschafter von ihrer Aufgabe, die sie zu einer asymmetrischen Einschätzung von Nutzen und Risiken verleitet. ${ }^{15}$ Tichy (2002) fand einen deutlich positiven ,insider bias' bei deutschen und österreichischen Einschätzungen längerfristiger Technologiepotentiale.

Zwangsläufig nährten die uneingelösten und uneinlösbaren Versprechungen der (Natur-)Wissenschafter Zweifel der Öffentlichkeit an der Objektivität der Wissenschaft; ${ }^{16}$ es entstand nicht bloß das dubiose Konzept des „Gegenexperten“ (Rucht 1988) ${ }^{17}$ - vielfach „,dissidente' Wissenschafter aus etablierten Wissenschafts- und Technikinstitutionen“ (v.d. Daele 1991, 40) -, sondern auch die Einsicht, dass die Wissenschaft nicht optimal geeignet ist, Gefahren herauszuarbeiten und abzuschätzen: ,[T] he conventional regulatory language of rationality (...) artificially reduces structural uncertainty and latent conflict to technical imprecision. Lack of control, deriving from ignorance and latent conflict, is concealed by the scientific language of manageable uncertainties" (Wynne 1987, ix, Hervorhebung im Original).

Mit dieser Erkenntnis war die Bahn frei für den Übergang zu einem sozialwissenschaftlichen ,Risiko'-Begriff: ${ }^{18}$ Evers und Nowotny (1987, 34ff) sehen als gesellschaftliches Risiko all das, was durch die öffentliche Diskussion ,aus der unbegrenzten Fülle von Handlungen, die mit Ungewissheit und möglichen Schäden verknüpft sein können - also aus dem Schattenreich der Gefahr - herausgeholt wurde, dass es durch gesellschaftliche Diskurse thematisiert und benennbar wurde, abgrenzbar und letztlich abwägbar. ... Gefahren werden gleichsam zivilisatorisch zugewiesen." Die Vorstellung der Öffentlichkeit von Sicherheit kommt in einem hochkomplexen Balancierungsprozess zustande (Evers und Nowotny 1987, 304), da sowohl die Gefahren (Douglas and Wildavsky 1982) als auch die Informationen (Thompson and Wildavsky 1982) durch kulturelle Filter wahrgenommen und in weiterer Folge akzeptiert oder abgelehnt werden; welche der vielen existierenden Gefahren zu ,Risiken' erklärt werden, bestimmt der gesellschaftliche Konsens. Entscheidend für die Fragestellung dieser Arbeit ist, dass die Institutionen des modernen Wohlfahrtsstaats Gefahren in gesellschaftlich anerkannte und regulierte ,Risiken' verwandeln können (Evers und Nowotny 1987, 70f).

14 Die Abschätzungen von zehn Teams betreffend die Wahrscheinlichkeit eines Störfalls im Kühlsystem einer Nuklearanlage differierten um einen Faktor 45; ein neuer Versuch unter Kenntnis der Ergebnisse der anderen Teams reduzierte die Differenz bloß auf einen Faktor 36. Die Festlegung einer einheitlichen Methode verminderte den Faktor auf 14, und erst die Vorgabe von einheitlicher Methode und einheitlicher Datenbasis ließ die Ergebnisse konvergieren.

15 Slovic (2000a, xxx) „observed a strong “affiliation bias' indicating that toxicologists who work for industry see chemicals as more benign than do their counterparts in academia and government."

16 Für den Bereich der Biotechnologie siehe Torgersen et al 2002, $61 \mathrm{f}$.

17 Der Begriff ist dubios, weil er zweierlei vernachlässigt: Erstens, dass die Fragen mit denen sich Wissenschaft beschäftigt noch nicht geklärt und insoweit strittig sind; insofern müssen Experten unterschiedlicher Meinung sein. Zweitens verschiebt sich nach Gibbons et al (1994) das disziplinäre Paradigma des „scientific discovery” (mode 1) allmählich zu dem der interdisziplinären „knowledge production” (mode 2), sodass Expertise zunehmend der Interdisziplinarität bedarf. Zu einer „Entgrenzung der Expertendiskussion“ (Rucht) kam es, als sich die ,Gegenexperten', Expertise' über Fragen fremder Fachgebiete anmaßten, und den fachlichen Diskurs mit alltagsweltlichen und machtpolitischen Fragen verknüpften, somit ihren Expertenstatus für Bewertungen missbrauchten, die sie als Staatsbürger abgaben.

18 Interessant ist, dass die Kritik nicht von der Wissenschaft sondern von der Politik ausging: 1956 hatte die Brookhaven-Studie (AEC 1957) mittels eines deterministischen Modells die Beherrschbarkeit eines AtomGAU bewiesen; der Congress, also Laien, drängte 1971/72 auf Anwendung probabilistischer Konzepte im sogenannten Rasmussen Report (AEC 1975); zur Kritik siehe Kollert (1993). 


\section{Die sinkende Risikoakzeptanz der Öffentlichkeit}

Der Gegensatz zwischen der ungewissheitsverdrängenden, technisierten Risikobewertung der Experten ${ }^{19}$ und der ungewissheitsfixierten, kontextvermittelten der Öffentlichkeit ist eine der wichtigsten Ursachen für den Trend steigender Verunsicherung: Die Einschätzung von Gefahren ${ }^{20}$ durch Experten weicht von der Wahrnehmung der Öffentlichkeit so weit ab, dass, die Wissenschaft' ihre Glaubwürdigkeit weitgehend eingebüßt hat; eine wichtige Orientierungshilfe in der zunehmend komplexen Welt ging damit verloren. Der Gegensatz musste aufbrechen, weil die üblichen ,Rationalitäts'-Kriterien des technischen Risikos für Laien wenig relevant sind. Der Wissenschafter lebt in seinem Fachbereich - und nur dort - in einer „Welt erschlossener, äußerst feiner Strukturen“ und „quantifizierter Regelmäßigkeiten“ (Slovic 2000b, xxiii), die Laien - trotz halbwegs realistischer Vorstellungen über das Schadenausmaß - in einer „Erfahrungswelt einzigartiger Ereignisse und mannigfaltiger Eigenschaften“, in einer „umgangssprachlich vorinterpretierten Lebenswelt“ (Huxley, zitiert nach Habermas 1968, 105; meine Hervorhebung G.T.); ihre Risikoeinschätzung beruht auf Alltagsheuristiken (Perrow [1987] 1989, 370f), ${ }^{21}$ die insofern unverzichtbar sind, als sie durch Vermeidung eines unendlichen Deliberationsprozesses Entscheidungen überhaupt erst ermöglichen, Suchkosten senken, und das Verhalten für andere vorhersehbar machen; sie funktionieren allerdings nur dann befriedigend, wenn sich das System bloß langsam ändert und lose gekoppelt ist; sie versagen demgemäss bei Strukturbrüchen.

Der Risikobegriff der Öffentlichkeit und die Kriterien für die Akzeptanz von Risiken wurden zunächst im Zusammenhang mit der Akzeptanz der Atomenergie diskutiert (Renn 1981); die Diskussion griff aber rasch auf andere Risken der Industriegesellschaft über. Die relevante Literatur auf den Gebieten der Psychologie, der Psychometrie, der Sozialpsychologie und der Soziologie ist inzwischen unübersehbar geworden. Es zeigte sich, dass die Öffentlichkeit sehr wohl zwischen unterschiedlichen Risken zu unterscheiden weiß und selbst innerhalb der Technologiefelder differenziert (TAB 2002). Anders als die Risikoeinschätzung der Experten, die mit dem technischen Risiko korreliert, sieht die Einschätzung der Laien von der Eintrittswahrscheinlichkeit weitgehend ab und orientiert sich an einer großen Zahl vielfach miteinander verbundener und zum Teil maßgeblich subjektiv geprägter Faktoren. Maßgebend sind vor allem die direkten und symbolischen Vorstellungen von der Gefahrenquelle, insbesondere ihr Katastrophenpotential („dread risk”, Slovic 1987, 283), das Angst- und Unbekanntheitsrisiko (Slovic et al zitiert bei Perrow 1989, 381f), die Neuheit der Technik, die Begleitumstände, insbesondere die Art des Auslösers bzw. der Information, die absolute Höhe des Schadens (Binswanger 1990, 108), der Zeitpunkt seines Eintritts sowie allgemeine Wertvor-

19 Typisch dafür die Aussage des Leiters des Electric Power Research Institute Palo Alto, California auf einer von General Motors veranstalteten Tagung: „The risk takers of the country are not ourselves but the great population of the country and they have perceptions of risk. Their perceptions may be so far from reality that you and I know that they're absurd, but that's how they feel about it and that's the way they perceive things" (Starr 1980, 4; meine Hervorhebung G.T.). Interessanterweise glauben Techniker jedoch insofern nicht an ihre eigenen Risikoabschätzungen, als sie über die unmittelbare Funktionstüchtigkeit hinausgehend systematisch zusätzliche Faktoren wie Sicherheitsnormen, Grenzwerte, Zuschläge etc. berücksichtigen (v.d. Daele 1991, 21), was zwar bei technischen, nicht aber bei Systemrisiken Sinn macht.

20 Die naturwissenschaftlich-technische Risikodebatte spricht auch in diesem Zusammenhang von ,Risiken'.

$21 \mathrm{Zu}$ diesen gehört etwa die Trennung von Informationsaufbereitung (,editing“) und Evaluierung, die Überbetonung aktueller Ereignisse oder die Bevorzugung sicherer Alternativen, von solchen mit kleinem sicheren Verlust, gegenüber solchen mit großem aber unsicheren Gewinn (Kahnemann and Tversky 1972; 1973; Camerer and Kunreuther 1989). 
stellungen, ${ }^{22}$ Persönlichkeitsmerkmale wie Alter und formale Schulbildung (Bechmann et al 1986) aber auch die gesellschaftliche Position. ${ }^{23}$ Besonders wichtig erscheint die Art der Darstellung der Gefahr, ${ }^{24}$ die Affektgeladenheit des Ereignisses, nicht zuletzt als Folge der Berichterstattung (Slovic [1997] 2000d, 404f), das Vertrauen in das Risikomanagement (Slovic [1993] 2000c), sowie die Position gegenüber dem Risiko: Freiwillig übernommene Risken werden erheblich geringer eingeschätzt (Slovic 1987, 282), ebenso Gefahren, die als persönlich kontrollierbar angesehen werden (Weinstein 1984).

Als wichtigstes Ergebnis der sozialwissenschaftlichen Diskussion ist festzuhalten, dass Risiko ein (soziales) Konstrukt ist (Douglas and Wildavsky 1982; Wiedemann 1993), und zwar ein prekäres Konstrukt (Evers und Nowotny 1987, 296). Warum aber erhielt dieses Konstrukt in den achtziger und neunziger Jahren , sensiblere Filter', warum wurde die Einstellung der Bürger zum Risiko kritischer, obwohl sie zumindest in Europa doch sicherer leben als früher? ${ }^{25}$ Ein wichtiges Element sind Zukunftsängste. Mag die Gegenwart auch sicher sein, die Zukunft erscheint gefährdet: Unzähligen kritischen oder gar apokalyptischen Stimmen zufolge bedrohen die Gefahren moderner Technologien die planetarische Zivilisation. ${ }^{26}$ Für Sachbuchautoren, Medien wie politische Ideologen ist Verunsicherung vielfach ein Element ihrer Marketingstrategie. ${ }^{27}$ Allerdings gibt es tatsächlich Argumente jenseits dieser Visionen, die ein zunehmendes Gefahrenpotential, vor allem an gesellschaftlich produzierten Gefahren, nicht unplausibel erscheinen lassen:

- Der technische und organisatorische Fortschritt ermöglicht die Transformation von Gefahren in Risiken, wodurch das Risiko ,angeschwollen' ist (Luhmann 1993, 328). ${ }^{28}$

- Viele der neuen Gefahren wirken global, mit oft irreversiblen Folgen (Jonas 1984; Lau 1989).

- Die Hochtechnologie ist generell nicht mehr voll beherrschbar und ein Systemversagen komplexer Technologien bedingt ein höheres Gefahrenpotential (Perrow 1986, sowie [1987] 1989); Komplexität impliziert vielfach „Effekterzeugung durch nicht-intendierte Effektkumulation (Luhmann [1984] 1987, 545).

22 ,[P] $[$ eople holding an egalitarian preference for wealth and power to be distributed equally in society had higher perceived risk for a wide range of hazards and were particularly concerned about nuclear power. People who prefer a hierarchical social order, in which experts and authorities are in control, had much lower perceptions of risk and more favourable attitudes toward nuclear power" (Peters and Slovic 1996 nach Slovic 2000b, xxxiii).

23 "[A]bout $30 \%$ of the white male sample ... judged risks to be extremely low." (Slovic [1997] 2000d, 398f). Dieses ,white male protestant"-Phänomen ergibt sich weitgehend daraus, dass höhere Schulbildung bessere Berufspositionen bedingt, und damit mehr aktive Entscheidung über Risiken und geringere Betroffenheit (Torgersen 2002, 175).

24 Z.B. als Sterbe- oder Überlebenswahrscheinlichkeiten (Tversky and Kahneman 1981; Slovic [1997] 2000d, 394).

25 Siehe etwa die kräftig steigende Lebenserwartung und die bessere Absicherung durch ausgebaute Sozialversicherung, steigende Privatversicherung und höhere Vermögen.

26 „Der Zukunftsschock“ (Toffler 1970), „Wachstumswahn und Umweltkrise“ (Commoner 1971), „Überlebensprognose“ (Vester 1972), „Ein Planet wird geplündert“ (Gruhl 1975), „Wege aus der Wohlstandsfalle“ (Binswanger et al 1978), „Wendezeit“ (Capra ${ }^{5}$ 1983), „Frieden mit der Natur“ (Meyer-Abich 1984), „Nulloption“ (Offe 1986), „Risikogesellschaft“ als „Katastrophengesellschaft“ (Beck 1986), „Gegengifte“ (Beck 1989), „Der Niedergang der modernen Welt"“ (Sinai 1990), ,Zeitalter der Extreme“ (Hobsbawn 1995).

27 Das betrifft keineswegs bloß ,alternative' Bewegungen; die neokonservativen Argumente einer ,Gefährdung des Standorts' durch mangelnde Deregulierung und das herrschende Lohn- und Sozialleistungsniveau oder der ,Unfinanzierbarkeit' der Sozialleistungen bedienen sich derselben Taktik.

28 „Wenn es Regenschirme gibt, kann man nicht mehr risikofrei leben: Die Gefahr, dass man durch Regen nass wird, wird zum Risiko, das man eingeht, wenn man den Regenschirm nicht mitnimmt. Aber wenn man ihn mitnimmt, läuft man das Risiko, ihn irgendwo liegen zu lassen“ (Luhmann 1993, 328). Ein ernsteres und aus heutiger Sicht relevanteres Beispiel bietet die pränatale Diagnostik: Sie transformiert die Unsicherheit ein ,geschädigtes' Kind zu bekommen in das Risiko der Entscheidung, eine solche Diagnose vornehmen zu lassen, sowie das Risiko einer Entscheidung bei ,negativem' oder unklarem Befund. 
- Sicherheitseinrichtungen erhöhen die Komplexität der Anlagen (LaPorte 1982) und lassen „safety risks" entstehen; in manchen Fällen können die Sicherheitsvorkehrungen die Gefahren sogar erhöhen (Wildavsky 1988, 48ff).

- Zusätzliche wissenschaftliche Untersuchungen reduzieren das Ausmaß der Unsicherheit nicht: Sie gehen von i. a. einem gegebenen (d. h. angenommenen) Risikensystem aus, können das Zusammenspiel der Elemente vielfach nicht modellieren, leiden unter „expert ignorance, structural uncertainty and objective conflict of perspectives and rationalities" (Wynne 1987, 421f).

- Viele Gefahren (z. B. Klimaerwärmung, Waldsterben) resultieren aus den Folgen einer Vielzahl alltäglicher Handlungen, die nicht leicht in den Griff zu bekommen sind und vielfach das Potential ,schleichender Katastrophen' haben.

- Bei manchen wissenschaftlich-technologischen Ansätzen, wie etwa bei manchen Anwendungen der Gentechnologie, besteht ein auffallendes Missverhältnis zwischen der Handlungsabsicht und möglichen Folgewirkungen.

- Das Bewusstsein stärkerer Bedrohung löste einen sich selbst verstärkenden Prozess aus: Wann immer ein Risiko auftritt, suchen Öffentlichkeit und Medien nach weiteren und sind dabei zumeist auch erfolgreich, was die Vorstellung unzähliger unentdeckter Bedrohungen und die hektische Suche nach diesen auslöst.

Die Häufung von Problemen auf so unterschiedlichen Gebieten wie Klima oder Pensionsfinanzierung hat weithin ein Bewusstsein von ,Grenzen' der bisherigen Entwicklung entstehen lassen, deren Überwindung tiefe Einschnitte erfordert und deren Folgen man von sich fernhalten möchte. Nicht bloß in den technischen Fortschritt ist das Vertrauen verloren gegangen, sogar stärker noch in die regulierenden Institutionen; subjektive Unsicherheit entstand, weil die Zukunft „,nicht mehr ausschließlich als positive Erweiterung bekannter Chancen und materiellen Wohlstands" gesehen wird (Evers und Nowotny 1987, 23). ${ }^{29}$ Die Wissenschaft wird nicht mehr als Bezwinger von Gefahren sondern vielfach sogar als deren Urheber gesehen; sie produziert nicht bloß Wissen sondern zugleich auch Nicht-Wissen und Noch-Nicht-Wissen, was besonders in Entscheidungssituationen zunehmend schmerzlich bewusst wird (Japp 1999). Demgemäss wird ,Sicherheit' von den Sozialwissenschaften zunehmend als Grundwert (Zapf 1987) und Gewährleistung von ,Sicherheit' als Staatsaufgabe (Ewald 1993) betrachtet. Die Volkswirtschaftslehre hat diese neue Zielvorstellung in ihren theoretischen wie in ihren wirtschaftspolitischen Ansätzen weitestgehend ignoriert, nicht zuletzt, weil - wie Abschnitt 2 gezeigt hat - ,Sicherheit' ein ,vorwissenschaftlicher Begriff ohne wissenschaftliche Potenz" ist, demgemäss auch nicht operationalisierbar und unmittelbar realisierbar. Einzelne Ansätze der sozialwissenschaftlichen Erklärung der Verunsicherung bieten dafür jedoch einen guten Ausgangspunkt; sie müssen kurz dargestellt werden, bevor die Folgen geänderter ,Risiko'-Vorstellungen für die wirtschaftliche Entwicklung herausgearbeitet werden können.

29 Siehe das Schlagwort von der, Vorbelastung der Zukunft und der künftigen Generation', sowie als unvermeidliche Reaktion auf diese floskelhaft rituell-folgenlos missbrauchte Formulierung: ,Was hat die Zukunft denn für mich getan? Respektiert sie meine Rechte?' Nach Perrow $(1989,104)$ schätzen zahlreiche Naturwissenschafter unter Berufung auf den technischen Fortschritt die Interessen der gegenwärtigen Generation tatsächlich höher ein. 


\title{
4 Sozialwissenschaftliche Ansätze zur Erklärung der zunehmenden Risikoaversion
}

\begin{abstract}
Anders als die Diskussion um die Formulierung eines sozialwissenschaftlichen ,Risiko'-Begriffs, die fast ausschließlich um technische und Umweltrisiken kreiste, setzt die sozialwissenschaftliche Erklärung der Verunsicherung sehr viel grundsätzlicher an. Im Zusammenhang mit der Erklärung der europäischen Stagnation genügt die Konzentration auf die drei zentralen Ansätze. ${ }^{30}$
\end{abstract}

Kaufmann $(1970,157)$ erklärt die zunehmende Verunsicherung, die Zunahme der empfundenen Gefahren, aus der „Schwierigkeit der Weltorientierung“ (ebd. 21): Wenn „Wertmaßstäbe außerhalb des Subjekts zerbrochen sind, wenn Wertmaßstäbe der Vergangenheit keine Verbindlichkeit mehr besitzen, muss eine Unsicherheit der Orientierung entstehen“ (ebd. 162). „Mit der Auflösung der die personale Identität der Individuen recht und schlecht garantierenden traditionalen Ordnungen wird die Konstituierung von Identität zu einem subjektbezogenen Problem“ (ebd. 250). „Es geht beim ,Sicherheitsgefühl' um die Wahrnehmung eines Gleichgewichts zwischen äußeren Risiken oder Ungewissheiten und inneren Möglichkeiten. Die beiden Formen ,äußerer Unsicherheit' - Gefahr und Ungewissheit - haben im Subjekt prinzipiell gleiche Wirkungen. Wenn dem so ist, so ist es kein Paradox, daß unter gesellschaftlichen Bedingungen, die - statistisch gesehen - das bisher höchste erreichte Maß an Gefahrlosigkeit für Leben und Gesundheit erreicht haben, Unsicherheit zur Zeitdiagnose wird“ (ebd. 313). „Wenn der Bereich des Möglichen zunimmt und derjenige des ,fraglos Gegebenen' abnimmt, so wirkt dies verunsichernd“ (ebd. 198).

Luhmann ([1968] ${ }^{4} 2000$, 60f) erklärt den Trend zunehmender Verunsicherung auf Grund systemtheoretischer Überlegungen als Verlust des Systemvertrauens: Die zunehmende Ausdifferenzierung von Wirtschaftssystem (Industrialisierung, Arbeitsteilung), politischem System (Demokratisierung, Wohlfahrtsstaat), Erziehungs-/Bildungssystem und Wissenschaftssystem (Kontrolle über die technischen Möglichkeiten) hat die funktionale Differenzierung der Gesellschaft geändert und komplexer gemacht. Hohe Komplexität der Welt setzt jedoch eine Vielzahl selektiver Prozesse voraus. Wenn der Einzelne diese Vielzahl nutzen möchte, bedarf es zunächst des persönlichen Vertrauens in die Urheber dieser Selektionen; bei längeren Selektionsketten wird jedoch der Rahmen persönlicher Bekanntschaften weit überschritten, es bedarf des Systemvertrauens (ebd. 64): man muss sich auf Informationsverarbeitung Unbekannter verlassen können. Es ,, ist eine Art von diffusem Gesamtvertrauen im Spiel“" (ebd. 69).

Luhmann vermutet, „dass das Altwerden eines bestimmten Differenzierungstypus Unsicherheit in Bezug auf die Zukunft erzeugt, die ihrerseits widerspruchsmultiplizierend wirkt. Die Ordnungsleistung wird selbstverständlich, ihre Mängel und Dysfunktionen treten auf Grund reicher Erfahrung schärfer hervor (Luhmann [1984] 1987, 516). In solchen Lagen wird die Zukunft in die Gegenwart hineingedrückt; man kommt nicht umhin, den Zukunftshorizont zu aktualisieren, ganz unabhängig vom konkret absehbaren Verlauf der Ereignisse. Dabei polarisieren sich die Ansichten über die $\mathrm{Zu}-$ kunft, positive und negative Utopien entstehen: Die neokonservativ-markteuphorischen High-techUtopien traten in den achtziger Jahren in scharfe Konfrontation zu den Horrorvisionen der Katastrophengesellschaft nach dem ,Zukunftsschock“.${ }^{31}$ Die ,wirtschaftlichen und politischen Eliten [versuchen stets] ... Risiken als integrale Bestandteile, wenn nicht als Motor der Fortschrittsspirale zu interpretieren. Gegen-Eliten ... sind ihrem Wesen nach ... daher immer Ergebnis der Konflikte um die soziale Kontrolle der dominierenden kulturellen Muster“" (Evers und Nowotny 1987, 45).

30 Für eine etwas breitere Darstellung siehe Tichy 2003b.

31 Siehe dazu auch Enquetekommission 1987. 
In dem dritten hier relevanten Ansatz erklären Evers und Nowotny (1987) die massive Verunsicherung der Bürger - an Hand des Vergleichs zweier Modernisierungsrisiken, Armut im 19. und Technisierung im 20. Jahrhundert - aus dem Ende des Aufholwachstums, dem Strukturwandel, der geänderten Nachfragestruktur, der Differenzierung der Einstellungen und zunehmend antagonistischen Vorstellungen über die Lösung der Probleme, gemeinsam mit externen Schocks: „Sicherheit im Sinne sozial vermittelter und damit geteilter Gewissheiten, Werte und Orientierungen“" (ebd. 45), in der Terminologie von Dahrendorf (1992) von Rollensicherheit und Bindungen, sei verloren gegangen. Man fürchte, die gewohnte Lebensqualität und den gewohnten Wohlstand durch die künftigen Folgen gegenwärtiger, Experimente' zu verlieren. Angst sei ,eine spezifische Form des Ausdrucks von Unsicherheit, ... ausgelöst durch Ohnmacht und Enteignungserfahrungen, oszillierend zwischen der Suche nach Selbstsicherheit und Zutrauen und dem heimlichen Festhaltenwollen an Sicherheit spendenden institutionellen Garanten“ (Evers und Nowotny 1987, 301). Diese institutionellen Garanten, also ,[i]nstitutionalisierte Sicherheitskomplexe, stehen in einem komplexen Wechselverhältnis zu anderen Formen der Sicherheit: gemeinschaftsbezogene Sicherheitsgaranten und individuell erworbene Kompetenzen. ... Wir bezeichnen sie als Selbstsicherheit“ (ebd. 61): Selbstsicherheit als souveräner Umgang mit dem, was als verbleibende Unsicherheit akzeptiert werden muss" (ebd. 302).

Evers und Nowotny stimmen mit Kaufmann überein, dass die Individuen diese Selbstsicherheit mit der Auflösung ihrer personalen Identität und dem Verlust der intersubjektiven Wertmaßstäbe eingebüßt haben; damit ist auch die ,Weltorientierung' verloren gegangen. Wenn aber ,wissenschaftliches Fachwissen und traditionelle Orientierungen im sozialen Wissen ihren früher gültigen Orientierungsrahmen verlieren, entstehen besonders tiefgreifende Verunsicherungen“ (ebd. 24). Sie können nur schwer überwunden werden, da Lernprozesse in Bezug auf Risiken nicht auf der Akkumulation von Wissen basieren, sondern auf dem Durchsetzungsvermögen von Akteuren und Bewegungen (Evers und Nowotny 1987, 28; Seifert 2002; Torgersen 2001; 2002): „Ein technischer Fortschritt der nicht mehr, passiert', sondern durch bewusste politische Akte gesteuert erscheint, wird politisch, und damit auch umstritten und legitimationspflichtig“" (Evers und Nowotny 1987, 188). Diese Legitimationspflicht kann derzeit in den großen EU-Staaten nicht eingelöst werden.

\section{Zunehmende Verunsicherung als Ursache der europäischen Stagnation}

Der sozialwissenschaftliche ,Risiko'-Begriff subsummiert unter ,gesellschaftlichem Risiko' all das, was die öffentliche Diskussion aus der unbegrenzten Fülle von Handlungen mit ungewissen Folgen und möglichen Schäden herausholt und durch Diskurs thematisiert und benennbar macht; insofern werden Gefahren zivilisatorisch zugewiesen. Die gesellschaftlichen Vorstellungen von ,Sicherheit' kommen in einem hochkomplexen Balancierungsprozess zustande, da Gefahren - wie auch Informationen - durch kulturelle Filter wahrgenommen werden. In der sozialwissenschaftlichen Debatte wurde zunächst die Verunsicherung der Gesellschaft durch die Industrialisierung thematisiert (Polanyi [1944] 1978), zu Beginn der siebziger Jahre die Gefahren des technischen Fortschritts (Großtechnologien) und der Umweltbedrohung. Als tiefer liegende Ursache wurde - wie Abschnitt 4 gezeigt hat - das Fehlen eines Orientierungsrahmens durch Verlust der intersubjektiven Wertmaßstäbe, der Zeitlichkeit der Zukunft und des Systemvertrauens herausgearbeitet; damit lässt sich sowohl das generelle Unbehagen an manchen Aspekten der Industriegesellschaft als auch der, Verunsicherungsschub' durch den Strukturbruch der siebziger Jahre und den Übergang von der „Sozialdemokratischen Geborgenheit zur Neuen Unsicherheit“ (Tichy 2004) recht gut erklären. Was der globale sozialwissenschaftliche Ansatz jedoch nicht in den Griff bekommt, ist einerseits die Unterbrechung 
des Trends steigender Verunsicherung durch Perioden geringer oder gar fehlender Verunsicherung - wie etwa die sechziger Jahre -, andererseits die erheblichen Länder-Unterschiede: Nicht überall prägt starke und zunehmende Verunsicherung das Klima, und die Wirtschaftslage differiert innerhalb der EU stärker als zwischen Europa und den USA. Wie Übersicht 1 zeigt, ist die Wirtschaftslage in den nordischen Staaten erheblich besser und die Verunsicherung viel geringer als in den drei großen EU-Mitgliedsstaaten. ${ }^{32}$ Das gilt für physische Sicherheit (Übersicht 1, Zeile 8$)^{33}$ und allgemeine Lebens-Zufriedenheit (Z. 5-7) ebenso wie für die Einschätzung von Veränderungen im allgemeinen und technischer Änderungen im Besonderen. Die größere Akzeptanz von Änderungen im Norden ist vor allem deswegen bemerkenswert, weil die Bevölkerung dort einen überdurchschnittlichen Einfluss der Veränderungen auf das eigene Leben erwartet (Z. 9), und das Gefühl mit dem technischen Fortschritt nicht Schritt halten zu können, keineswegs schwächer ausgeprägt ist (Z.10); dennoch werden diese Änderungen akzeptiert (Z. 11) und sogar als Chance wahrgenommen (Z. 12). Auch neuen Technologien stehen die Skandinavier in jeder Weise positiver gegenüber als Deutsche, Franzosen und Italiener (Z. 13-19).

Das hohe Ausmaß an Vertrauen und positiver Einstellung in Skandinavien überrascht insofern, als Deregulierung und ,Sozialabbau' in Skandinavien stärker forciert wurden als in den großen stagnierenden Volkswirtschaften, sodass eigentlich größere Verunsicherung zu erwarten gewesen wäre. Die überdurchschnittliche Deregulierung der skandinavischen Gütermärkte lässt sich zwar in den Gesamtindikatoren weniger klar erkennen (Übersicht 2, Z.1), weil weniger privatisiert wurde; sehr markant zeigt sie sich aber in geringeren Eingriffen in das operative Geschäft und in schwächerer administrativer Regulierung. In dieser Hinsicht nehmen die großen drei europäischen Flächenstaaten die letzten Plätze ein. Bei der Arbeitsmarktregulierung zeigen die Noten des Gesamtindikators der OECD in Skandinavien eine um gut eine Notestufe stärkere Liberalisierung. Die Zumutbarkeitsbestimmungen in der Arbeitslosenversicherung sind in allen skandinavischen Staaten viel strikter, allerdings auch die Einkommensersatzraten höher (Nickell 2003, Tab. 4 und 6). Die Steuerbelastung wurde in den neunziger Jahren im Norden gesenkt, in Deutschland und Frankreich erhöht, die Sozialquote in den skandinavischen Ländern etwa gleichgehalten, in allen drei wachstumsschwachen großen Ländern z. T. markant erhöht. Die Evidenz erscheint somit zunächst weder mit den Thesen der Volkswirte noch mit denen der Sozialwissenschafter vereinbar: Einerseits hätte im Norden das hohe Niveau von Steuer- und Sozialquote die wirtschaftliche Entwicklung bremsen, andererseits deren tendenzieller Abbau und die Deregulierung verunsichernd wirken müssen; in den großen Drei wäre die gegenteilige Entwicklung zu erwarten gewesen.

Eine mögliche Erklärung des Paradoxons stärkerer Verunsicherung in den Stagnationsländern trotz Verzicht auf unbeliebte Änderungen wie Deregulierung und Sozialabbau (bzw. Mangel an Verunsicherung trotz Liberalisierung in Skandinavien) könnte im induzierten Wachstum liegen: Deregulierung, Reduzierung der Staats- und Limitierung der Sozialausgaben im Norden hätten Wirtschaftsund vor allem Produktivitätswachstum gesteigert (siehe Zeilen 1-2 in Übersicht 1) und das Bewusstsein dieser Erfolge die Verunsicherung überwunden. Das Argument kann aus zwei Gründen nicht voll überzeugen: Erstens, weil das raschere Wachstum in Skandinavien auf die für die Bewusstseinsbildung besonders relevanten Größen Beschäftigung und Reallohnsteigerung keineswegs voll durchgeschlagen hat. Zweitens, weil die Bevölkerung im Norden das raschere Wachstum keinesfalls euphorisch einschätzt: Sie ist sich bewusst, dass es mit unangenehmen Einflüssen auf das persönliche Leben verbunden ist; der technische Fortschritt erscheint auch hier einem Viertel bis einem Drittel der Bevölkerung zu rasch (Übersicht 1), und sein Einfluss auf die künftige Lebensqualität und die künftige Sicherheit wird kaum günstiger beurteilt als in den drei großen Staaten.

32 Die ungünstigen Werte für Deutschland sind nicht Folge der Einbeziehung der neuen Länder; die alten Bundesländer allein würden in allen Fällen denselben Platz in der Rangordnung der EU-Mitglieder einnehmen.

33 Der Indikator mag zu spezifisch erscheinen, doch erweist er sich empirisch als guter Indikator generellen Vertrauens (Delhey and Newton 2002, 21). 
Die schwache Evidenz für eine Erklärung der geringeren Verunsicherung aus der günstigeren Wirtschaftsentwicklung legt die Vermutung nahe, dass genau umgekehrt geringere Verunsicherung die Entscheidungen der Investoren, der Innovatoren und der Konsumenten positiv beeinflusst und dadurch das Wachstum forciert hat: Als Folge ihrer geringeren Verunsicherung hielten die skandinavischen Haushalte ihr Konsumniveau auch in Perioden schwächeren Wachstums und finanzierten es durch Absenkung ihrer Sparquote (Übersicht 3); die skandinavischen Investoren investierten zwar nicht mehr als diejenigen in den drei großen Ländern, doch sie investierten offensiv und zukunftsgerichtet: Statt in traditionelles physisches Kapital stärker in moderne Bereiche wie Informationsund Kommunikationstechnologie, sowie in Humankapital; die Innovationen profitierten in Skandinavien von der höheren F\&E-Quote der Unternehmen und der besseren Ausbildung der Bevölkerung (Übersicht 3). Im Zentrum der EU hingegen zwang die Verunsicherung die Konsumenten mehr zu sparen, und die Unternehmen investierten bloß zur Rationalisierung und schränkten ihre F\&EAusgaben ein.

Übersicht 1: Wirtschaftslage und Verunsicherung

\begin{tabular}{|c|c|c|c|c|c|c|c|}
\hline & & DK & $\mathrm{S}$ & FIN & $\mathrm{D}$ & $\mathrm{F}$ & 1 \\
\hline \multicolumn{8}{|c|}{ Wirtschaftslage } \\
\hline \multicolumn{2}{|c|}{ I. GDP-Wachstum 1993/2002 } & 2,5 & 2,9 & 3,3 & 1,3 & 1,9 & 1,6 \\
\hline \multicolumn{2}{|c|}{ 2. Veränderung der totalen Faktorproduktivität 1993/2002 } & $\mathrm{I}, 6$ & 2,4 & 2,7 & 0,4 & 0,9 & 0,8 \\
\hline \multicolumn{2}{|c|}{ 3. Arbeitslosenquote 1993/2002 } & 5,8 & 7,7 & 12,5 & 8,4 & 10,7 & 10,8 \\
\hline \multicolumn{2}{|c|}{ 4. Reallohnsteigerung $1993 / 2002$} & $1 / 2$ & $21 / 4$ & $21 / 2$ & I & $11 / 2$ & $-1 / 4$ \\
\hline \multicolumn{8}{|c|}{ Verunsicherung $(200 \mathrm{I})$} \\
\hline \multicolumn{2}{|c|}{ 5. Lebenszufriedenheit: \% sehr zufrieden } & 64 & 37 & 25 & 14 & 13 & 12 \\
\hline \multicolumn{2}{|r|}{ nicht zufrieden } & 3 & 7 & 9 & 21 & 21 & 19 \\
\hline \multicolumn{2}{|c|}{ 7. Lebenszufriedenheit in 5 Jahren: \% sehr zufrieden } & 40 & 48 & 37 & 20 & 42 & 53 \\
\hline \multicolumn{2}{|c|}{$\begin{array}{l}\text { 8. Etwas/sehr unsicher bei Spaziergang in Wohngegend } \\
\text { bei Dunkelheit }\end{array}$} & 15 & 21 & 19 & 33 & 34 & 42 \\
\hline \multicolumn{2}{|c|}{$\begin{array}{l}\text { 9. Gibt es wichtige Änderungen in der Welt die Sie } \\
\text { persönlich betreffen }\end{array}$} & 58 & 65 & 66 & 47 & 42 & 60 \\
\hline \multicolumn{2}{|c|}{ 10. Ich mag diese Art von Änderungen nicht } & 8 & 10 & 10 & 17 & 11 & 16 \\
\hline \multicolumn{2}{|c|}{$\begin{array}{l}\text { II. Die Änderungen erfolgen so rasch, dass ich ihnen kaum } \\
\text { folgen kann }\end{array}$} & 22 & 30 & 32 & 20 & 26 & 31 \\
\hline \multicolumn{2}{|c|}{$\begin{array}{l}\text { 12. Die Änderungen bieten mir Chancen und erleichtern mein } \\
\text { tägliches Leben }\end{array}$} & 52 & 38 & 36 & 32 & 30 & 29 \\
\hline \multicolumn{2}{|c|}{$\begin{array}{l}\text { 13. Neue Technologie nächste } 5-10 \text { J:: } \\
\text { Macht Leben schwerer \& erhöht Stress }\end{array}$} & 5 & 14 & 5 & 15 & 13 & 10 \\
\hline \multicolumn{2}{|r|}{ leichter \& senkt Stress } & 38 & 30 & 32 & 25 & 23 & 24 \\
\hline 15. & Verringert Sicherheitsgefühl & 22 & 30 & 31 & 39 & 34 & 23 \\
\hline 16. & Erhöht Sicherheitsgefühl & 14 & 29 & 27 & 20 & 17 & 30 \\
\hline \multirow{2}{*}{$\begin{array}{l}17 . \\
18 .\end{array}$} & Einfluss auf Familie\& Sozialbeziehungen + & 56 & 53 & 42 & 29 & 37 & 45 \\
\hline & - & 10 & 16 & 15 & 21 & 14 & 17 \\
\hline \multicolumn{2}{|l|}{20.} & 29 & 26 & 38 & 33 & 42 & 26 \\
\hline
\end{tabular}

Quelle: Aiginger 2003b; Eurobarometer 58.0 (Autumn 2002), 54.2 (February 2001) 
Übersicht 2: Deregulierung

\begin{tabular}{|c|c|c|c|c|c|c|}
\hline & DK & S & FIN & $\mathrm{D}$ & $\mathrm{F}$ & I \\
\hline Gütermarktregulierung* (1998) & $\mathrm{I}, 4$ & 1,4 & 1,7 & 1,4 & 2,1 & 2,3 \\
\hline Staatliche Kontrolle & 2,5 & 1,5 & 2,7 & 1,8 & 2,6 & 3,9 \\
\hline Öffentliches Eigentum & 2,3 & 2,3 & 3,3 & 1,2 & 2,3 & 4,4 \\
\hline Eingriffe in das operative Geschäft & 2,7 & 0,6 & 1,9 & 2,5 & 3,0 & 3,3 \\
\hline Administrative Regulierung & $\mathrm{I}, \mathrm{I}$ & 2,0 & 2,2 & 2,7 & 3,1 & 3,0 \\
\hline \multirow[t]{3}{*}{ Arbeitsmarktregulierung* (1998) insgesamt } & 1,5 & 2,4 & 2,1 & 2,8 & 3,1 & 3,3 \\
\hline & $\mathrm{I}, 7$ & 3,0 & 2,3 & 3,0 & 2,5 & 3,0 \\
\hline & 1,2 & 1,8 & 1,9 & 2,5 & 3,7 & 3,6 \\
\hline Zumutbarkeitsbestimmungen (Arbeitslosenversicherung) & 3,0 & 3,7 & 2,7 & 2,6 & 2,7 & - \\
\hline Einkommensersatzrate \% & 66 & 74 & 54 & 37 & 59 & - \\
\hline Steuern/GDP (Veränderung 1993/2002 in \%-Punkten) & $-0,8$ & $-5,4$ & $-7,3$ & $+1,4$ & $+2,3$ & $-0,6$ \\
\hline Sozialausgaben/GDP (Veränderung 1990/2002 in \%-Punkten) & $+0,1$ & $-0,8$ & $+0,1$ & $+4,1$ & $+1,8$ & $+0,5$ \\
\hline
\end{tabular}

* 1 niedrig, 6 hoch

Quelle: Nicoletti et al 2000; Nickell 2003; Aiginger 2003b; Wifo-Datenbank

Übersicht 3: Stabilisierende Faktoren

\begin{tabular}{lccc:ccc}
\hline & DK & S & FIN & D & F & I \\
\hline Steuern in \% des GDP 2002 & 57 & 59 & 54 & 45 & 51 & 45 \\
Staatsausgaben in \% des GDP 2002 & 55 & 58 & 49 & 49 & 54 & 48 \\
Sozialausgaben in \% des GDP 2002 & 29 & 32 & 25 & 30 & 30 & 25 \\
& & & & & & \\
Private Sparquote (in \% des verf. Einkommens) 200I & $-11 / 4$ & $21 / 2$ & 0 & $101 / 4$ & $111 / 2$ & 10 \\
Private Investitionen/GDP 200I & 17,3 & 13,4 & 13,0 & 12,7 & 12,8 & 13,7 \\
& & & & & & \\
Private Investitionen/GDP 1992/99 & 13,5 & 10,8 & 10,9 & 12,6 & 11,2 & 11,6 \\
F\&E-Ausgaben/GDP 1992/99 & 1,8 & 3,4 & 2,5 & 2,4 & 2,3 & 1,2 \\
F\&E-Ausgaben der Unternehmungen/GDP 1992/99 & 1,1 & 2,5 & 1,6 & 1,6 & 1,4 & 0,6 \\
IKT-Ausgaben/GDP I992/99 & 5,1 & 5,0 & 6,1 & 4,6 & 4,6 & 3,7 \\
Anteil der 25-64-jährigen mit tertiärer Ausbildung & 26 & 32 & 32 & 23 & 23 & 10 \\
\hline
\end{tabular}

Quelle: Wifo-Datenbank; OECD

Was aber erklärt die offensichtlich geringere Verunsicherung im Norden? Ein Element dürfte die stärkere staatliche Absicherung sein: Staatsausgaben wie Steuern, und bis vor kurzem auch die Sozialausgaben, sind im Norden deutlich höher (Übersicht 3). Eine solche Absicherungsstrategie ist keineswegs neu: Katzenstein $(1985,47)$ hat schon vor zwei Jahrzehnten darauf hingewiesen, dass einige kleinere europäische Staaten die aus ihrer liberalen Außenwirtschaftspolitik resultierenden Risken durch Maßnahmen der Investitions- und Industriepolitik sowie der Einkommens- und Transferpolitik absichern. Rodrik (1998) zeigte jüngst, dass eine derartige Kompensationsstrategie nicht bloß in den europäischen Kleinstaaten, sondern weltweit betrieben wird: ${ }^{34}$ Die Ausgaben für So-

34 Seine Länderstichprobe umfasst je nach Datenverfügbarkeit etwa 80 bis 150 Länder. 
zialtransfers (in den Industriestaaten) und für Staatskonsum (in den weniger entwickelten Ländern) sind desto höher, je größer das Risiko im Außenhandel ist, gemessen an der Varianz der Terms of Trade und der Produkt-Konzentration. Rodrik kann auch zeigen, dass eine solche Politik erfolgreich ist: In 119 von 147 Ländern ist die stabilisierende Wirkung signifikant.

Der Absicherungs-Ansatz von Katzenstein und Rodrik kann somit die Unterschiede im Verunsicherungsgrad zwischen Norden und Zentrum aus dem Niveau der staatlichen Absicherung erklären. Allerdings hat das Niveau, wie erwähnt, in den nordischen Staaten abgenommen, und überdies haben die meisten dieser Volkswirtschaften erhebliche Anpassungen, vielfach sogar in der Form von Krisen hinter sich; in den drei großen Flächenstaaten hingegen hat die Absicherung im Wege höherer Staats- und Sozialquote im letzten Jahrzehnt eher zugenommen und größere (Struktur-)Anpassungen fehlen zumeist. Wieso wirkte die Reduzierung der Absicherung im Norden nicht verunsichernd, deren Ausweitung im Zentrum nicht beruhigend?

\section{Mangelndes Systemvertrauen in den großen EU-Staaten}

Geht man mit Luhmann ([1968] ${ }^{4} 2000$, 60ff) davon aus, dass es bei zunehmender Komplexität der Gesellschaft $^{35}$ eines Vertrauens in das System bedarf, „einer Art von diffusem Gesamtvertrauen“, mit dem man sich auf die Informationsverarbeitung Unbekannter verlässt, weil man selbst die Folgen der unterschiedlichen Optionen nicht abschätzen kann (siehe Abschnitt 4), spricht Manches dafür, dass einer der wichtigsten Sicherheit-schaffenden Faktoren eine konsistente und konsequente (Wirtschafts-)Politik ist, die auf die psychologischen Folgen ihrer Maßnahmen achtet. Gerade zentrale wirtschafts- und sozialpolitische Fragen - Deregulierung, Flexibilisierung, Pensionsreform, Gesundheitsreform, Reformstau etc. - stecken jedoch in den stagnierenden Großstaaten zugleich in einer verunsichernden, hektischen Medien-Debatte und in einem hartnäckigen Reformstau; gerade in diesen Bereich dürften auch die kulturellen Filter, durch die Gefahren und Informationen wahrgenommen und eingeschätzt werden, besonders wichtig sein und der Balancierungsprozess der Bildung von Sicherheitsvorstellungen besonders komplex.

Tatsächlich scheinen in der Art der Wirtschaftspolitik und ihrer Einschätzung durch die Betroffenen die größten Unterschiede zwischen den Staaten des Nordens und denen des Zentrums zu liegen. Im Norden setzte man auf Konsens und ausgewogene Maßnahmenbündel: Zwar wurden Staatsausgaben- und Sozialquote gesenkt, ihr Niveau blieb aber relativ hoch; der Arbeitsmarkt wurde dereguliert und die Zugangsbestimmungen zur Arbeitslosenunterstützung verschärft, doch stärker im Bereich der temporären Verträge und begleitet von hohen Einkommensersatzraten, aktiver Arbeitsmarktpolitik und einem Ausbau der Schulungsmaßnahmen (Nickell 2003, 19f; Kaufmann 2003, 306). Die Wirtschafts- und Sozialpolitik wird im Norden zumeist längerfristig geplant und relativ konsequent durchgezogen.

Übersicht 4 lässt die Unterschiede im Systemvertrauen und einzelner seiner konstituierenden Elemente klar erkennen: Das Vertrauen der Skandinavier untereinander ist rund doppelt so hoch wie

35 In diesem Zusammenhang ist irrelevant, ob die Komplexität tatsächlich hoch ist bzw. zunimmt, oder ob das die Betroffenen bloß so empfinden. 
das der Bevölkerung der großen Flächenstaaten, das Vertrauen in den Staat um ein Viertel höher. ${ }^{36}$ Die Beschäftigung gilt als sicherer, obwohl etwa die Hälfte der Skandinavier angibt, ihren Arbeitsplatz in den letzten fünf Jahren gewechselt zu haben (in den drei großen Staaten im Zentrum Europas bloß ein Viertel); und verglichen mit den drei stagnierenden Staaten glauben gut doppelt so viele Skandinavier ihn in den nächsten fünf Jahren zu wechseln. ${ }^{37}$ Dahinter steht ein Ausbildungs- und Organisationssystem der skandinavischen Firmen, das rund doppelt so viele Mitarbeiter betrieblich weiterbildet und stärker Verantwortung delegiert. ${ }^{38}$ Dahinter steht aber auch eine stärkere Umverteilung durch den Staat: Die Verteilung der Markteinkommen in Skandinavien entspricht etwa der in den drei großen Staaten, die Umverteilung ist erheblich stärker; sie wurde in den neunziger Jahren sogar weiter forciert, wogegen sie in den großen Drei reduziert wurde (Schettkatt 2003, 29).

Übersicht 4: Elemente des Vertrauens und der Vertrauensbildung

\begin{tabular}{lccc:ccc}
\hline & DK & S & FIN & D & F & I \\
\hline „Trust“ (den meisten Leuten kann man vertrauen) \% & 56 & 57 & 57 & 30 & 25 & 26 \\
„Norms of civic cooperation” \% & 40 & 42 & $4 I$ & 40 & 36 & 4 I \\
Vertrauen in die Regierung \% & 76 & 65 & 66 & 54 & 62 & 44 \\
& & & & & & \\
Arbeitsplatzsicherheit Männer (niedrig = sicher) & 1,6 & 2,2 & 2,1 & 2,2 & 2,4 & 2,2 \\
Kein Arbeitsplatzwechsel in den letzten 5 Jahren & 47 & 56 & $6 I$ & 78 & 73 & 77 \\
Arbeitsplatzwechsel in den nächsten 5 Jahren & 38 & 37 & 33 & 10 & 19 & 15 \\
& & & & & & \\
Anteil der Beschäftigten ohne Firmenfortbildung 200I & 22,9 & 34,2 & 26,2 & 44,5 & 67,1 & 62,0 \\
Anteil mit verbesserter Qualifikation 200I & 49,5 & 44,5 & 65,7 & 40,8 & 42,2 & 47,0 \\
Anteil mit erhöhter Verantwortung 200I & 50,1 & 56,2 & 56,9 & 45,9 & 47,0 & 48,4 \\
& & & & & & \\
Einkommen vor Umverteilung I990er J. (Ginikoeff.) & 0,36 & 0,42 & 0,38 & 0,37 & 0,41 & 0,46 \\
Einkommen nach Umverteilung I990er J. (Ginikoeff.) & 0,21 & 0,25 & 0,24 & 0,28 & 0,28 & 0,34 \\
Umverteilung (Differenz) & $-0,15$ & $-0,17$ & $-0,14$ & $-0,09$ & $-0,13$ & $-0,12$ \\
\hline
\end{tabular}

Quelle: Knack and Keefer 1997, 1285; Eurobarometer 54.2, Schettkatt 2003, 29.

Die empirische Evidenz spricht für die Hypothese, dass die geringere Verunsicherung in Skandinavien die Voraussetzung für die Deregulierung und die Reform des Sozialsystems bildete. Cassel (1900, zitiert nach Agell 2003) betonte schon zu Beginn des vorigen Jahrhunderts, dass die Sozialpolitik insofern ein machtvolles Instrument zur Förderung der wirtschaftlichen Entwicklung wäre, als sie die Bereitschaft zur Akzeptanz des Wandels erhöht. Sozialpolitik kann dazu beitragen, dass die unvermeidliche „Aktualisierung des Zukunftshorizonts“ (Luhmann) weniger polarisierende idealtypische Utopien auslöst, und „Sicherheit im Sinne sozial vermittelter und damit geteilter Gewissheiten, Werte und Orientierungen“ (Evers und Nowotny 1987, 45) weitgehend erhalten bleibt. Zum Sicherheitsgefühl der Nordländer trug neben der Sozialpolitik auch die konsequente Wachstumspolitik bei, die den technischen Fortschritt in einem günstigeren Licht, nämlich als Chance, er-

36 Bjørnskov (2003) stellt eine signifikante Beziehung zwischen dem Vertrauen (in seiner Terminologie ,social capital") und der Lebenszufriedenheit fest.

37 Die Bereitschaft zum Arbeitsplatzwechsel mag auch dadurch stimuliert werden, dass Arbeitslosigkeit in Skandinavien weniger negativ einschätzt wird und die Arbeitslosen weniger stigmatisiert sind als etwa in Deutschland (DIE ZEIT 41, 1.10.2003, 30f).

38 Freeman $(1998,9)$ konnte zeigen, dass sich Firmen mit employee participation günstiger entwickeln. 
scheinen lässt: Forschungsquote und Bildungsausgaben sind überdurchschnittlich hoch und es gibt effiziente Einrichtungen der Technologiepolitik (Aiginger 2003b).

Ein positiver Einfluss des Systemvertrauens auf die wirtschaftliche Entwicklung entspricht durchaus den Erkenntnissen der sozialwissenschaftlichen Forschung und wurde etwa von Knack and Keefer (1997) für eine Stichprobe von 29 Ländern empirisch nachgewiesen. Zugleich zeigte diese Studie auch, dass das Systemvertrauen in denjenigen Ländern stärker ausgeprägt ist, in denen die Einkommen höher und gleichmäßiger verteilt sind. Das bestätigt die Vermutung, dass es der skandinavischen Politik, die den Wohlfahrtsstaat zwar nie grundsätzlich in Frage stellte, ihn aber behutsam reformierte, gelang, die Verunsicherung aufzufangen, die aus der zunehmenden Komplexität und der generell sinkenden Selbstsicherheit resultiert. Im Gegensatz dazu prägt das Schlagwort vom Reformstau die Diskussion der großen Länder: Radikale und weitreichende Reformkonzepte werden laufend diskutiert, bestenfalls teilweise verwirklicht, und vielfach wieder zurückgenommen; Abbau der Staats- und Sozialquote, Liberalisierung und Flexibilisierung des Arbeitsmarkts und Reduzierung der Rechte der Arbeitslosen - also Reduzierung aller Absicherungselemente zugleich - werden gefordert und vorbereitet, können politisch aber bestenfalls zum Teil durchgesetzt werden: Die Unverzichtbarkeit einer drastischen Senkung von Staats- und Sozialquote für ,Standort' und ,Zukunftsfähigkeit' wird gepredigt, de facto steigen beide. Der Sanierungserfolg bleibt aus, die Verunsicherungs-Wirkung tritt gerade deswegen voll ein. Wenn für die Bürger nicht bloß in ihrem persönlichen Leben sondern auch im Bereich der Wirtschafts- und Sozialpolitik „der Bereich des Möglichen [und zwar primär in für sie negative Richtung, G.T.] zunimmt und derjenige des ,fraglos Gegebenen' abnimmt, so wirkt dies verunsichernd“ (Kaufmann 1970, 198); die Betroffenen fürchten die gewohnte Lebensqualität, den gewohnten Wohlstand und das gewohnte Sicherheitsnetz durch die künftigen Folgen geplanter wirtschafts- und gesellschaftspolitischer ,Experimente' zu verlieren: Angst entsteht als ,,spezifische Form des Ausdrucks von Unsicherheit, ... ausgelöst durch Ohnmacht und Enteignungserfahrungen“" (Evers und Nowotny 1987, 301). ${ }^{39}$

Die Erkenntnis, dass die Verunsicherung der Bevölkerung die europäische Stagnation zumindest mitverursacht, steht im Gegensatz zu den meisten internationalen Studien, vor allem solchen von OECD, Währungsfonds und EU-Kommission, die dafür ausschließlich den hohen Staatsanteil, die hohen Sozialkosten und die geringe Flexibilität der Märkte verantwortlich machen. Tatsächlich zeigt der Vergleich der rasch wachsenden skandinavischen mit den stagnierenden Volkswirtschaften der großen europäischen Länder, dass Staatseigentum (Übersicht 2), Steuerquote (Übersicht 2) und Umverteilung (Übersicht 4) zumindest in Skandinavien keine Wachstumshindernisse bilden; Deregulierung der Märkte scheint relevant zu sein, doch muss sie in Formen erfolgen, die das Vertrauen der Bevölkerung und ihr Sicherheitsbedürfnis nicht beeinträchtigen. Das legt die Schlussfolgerung nahe, dass die Wirtschaftspolitik beim Abbau selbst kontraproduktiver Regulierung und überhöhter Transferausgaben sehr geschickt und mit Hilfe von systemkonformen Maßnahmenbündeln agieren muss, um verunsichernde Nebenwirkungen auf Konsum und Investitionen zu vermeiden.

Die skandinavische Erfahrung zeigt, dass Europa keineswegs hinter die US-amerikanischen Wachstumsraten zurückfallen muss. Die unterschiedlichen Institutionen und die unterschiedliche Mentalität (Tichy 2003a) erfordern allerdings andere Konzepte. In Skandinavien dürfte eine gut abgestimmte und glaubwürdige Kombination von Bildungspolitik, Technologiepolitik, Sozialpolitik und maßgeschneiderter Deregulierung die entscheidende Rolle gespielt haben; mit ihrer Hilfe gelang es, den Trend steigender Verunsicherung trotz der unvermeidlichen Änderung der Institutionen in Grenzen zu halten.

39 „Und wir haben den Eindruck, kaum noch zwischen Chancen wählen zu können, sondern uns immer öfter zwischen Übeln entscheiden zu müssen. ... Ob bei Bildung, Gesundheit, Rente, der Staat gibt Verantwortung ab. Damit werden die Risken und die Zukunft zunehmend individualisiert" (U. Beck im Interview in DIE ZEIT 33, 7.8.2003, 43). 


\section{Die Vernachlässigung der ,Risiko'-Diskussion durch die Volkswirte}

Die sozialwissenschaftliche Diskussion der letzten Jahrzehnte hat zu einer ersten Klärung der Begriffe geführt: Der Begriff der Sicherheit wurde als undefinierbar und wissenschaftlich unbrauchbar ausgeschieden. Als Gefahr werden solche Schäden bezeichnet, deren Eintritt gegenwärtig unsicher und mehr oder weniger unwahrscheinlich ist und der Umwelt zugerechnet wird. Risiken sind gleichfalls Schäden, deren Eintritt gegenwärtig unsicher und mehr oder weniger unwahrscheinlich ist; die sozialwissenschaftliche Definition von Risiko betont - als Korrelat zur Definition der Gefahr -, dass der Schadenseintritt als Folge eigenen Handelns oder Unterlassens gesehen wird, sowie dass den Risiken, anders als den Gefahren, auch Chancen inhärent sind. Die versicherungstechnische wie die volkswirtschaftliche Definition von Risiko stellt hingegen auf die Abschätzbarkeit des Schadenseintritts auf Grund des Gesetzes der großen Zahl ab. Die beiden Definitionen erscheinen als durchaus kompatibel: In der ersten Definition wird der Mikroaspekt betont - aus dieser Sicht wird z. B. das Ableben vom einzelnen Betroffenen, da von ihm i. a. nicht beeinflussbar, als Gefahr gesehen; in der zweiten Definition erscheint es in Makrosicht als Risiko, da die Lebenserwartung statistisch gut abschätzbar ist, man sich somit zwar nicht gegen den ,Schaden' des Ablebens, wohl aber gegen manche seiner Folgeschäden versichern kann. Das Abstellen der Definition auf die jeweilige Sichtweise erscheint nicht zuletzt deswegen zweckmäßig, weil der technische bzw. organisatorische Fortschritt vielfach Entscheidungsmöglichkeiten schafft, Gefahr in Risiko zu transformieren - etwa durch die ,Erfindung' der Lebensversicherung oder der pränatalen Diagnostik. Noch wichtiger als die Klärung der Begriffe erscheint jedoch die Erkenntnis, dass Risiken wie Gefahren soziale Konstrukte sind und demgemäss durch Entscheidungen (Anderer) ,sozial erzeugt' werden.

Die Nationalökonomie hat diese sozialwissenschaftlichen Erkenntnisse weitgehend ignoriert; im Modell der Rationalen Erwartungen wird jede Ungewissheit zwangsläufig zum Risiko. Sie befindet sich damit in guter Gesellschaft, denn auch die naturwissenschaftlich-technologiepolitische Diskussion definiert Gefahren in Risken um, indem sie die versicherungstechnische Risikodefinition - mangels genügender Fallzahlen unzulässigerweise - auf Gefahren überträgt (etwa in der Diskussion um Atomgefahren); zugleich besteht sie - entgegen aller empirischen Erfahrung ${ }^{40}$ - darauf, dass die Gefahrenangst der Öffentlichkeit auf mangelndem Wissen beruhe, sodass Akzeptanz durch Kommunikation von Wissen erreicht werden könne.

Die Volkswirte könnten von einer intensiveren Beschäftigung mit den sozialwissenschaftlichen Erkenntnissen betreffend Gefahren, Risiko und die Folgen von Verunsicherung erheblich profitieren, vor allem von den unterschiedlichen Folgen von Gefahr und Risiko für Erwartungsbildung und Entscheidungen. So etwa könnte die Berücksichtigung der Ergebnisse der oben zitierten Analysen von Kaufmann, Luhmann oder Evers und Nowotny - wie zu zeigen versucht wurde - nicht bloß zur Erklärung längerfristiger Stagnationsperioden beitragen, sondern auch zum Entwurf wirtschaftspolitischer Konzepte. Zeigen sie doch deutlich, dass die aus Veränderungen (Strukturbrüchen) resultierende Verunsicherung desto größer ist, je mehr das Vertrauen in das System geschwunden ist; nicht zufälligerweise ist die Verunsicherung durch den Strukturbruch in erster Linie in den Gesellschaften problematisch, in denen der Mythos der Sicherheit und Machbarkeit besonders ausgeprägt ist (Evers und Nowotny 1987, 301). ${ }^{41}$ Die Systemkonformität von Maßnahmen wurde von den Volks-

40 Gaskell et al (forthcoming).

41 Siehe etwa die relativ zu den USA sehr viel größere Verunsicherung durch Gentechnik in Deutschland, oder die Unterschiede in der Risikoeinschätzung zwischen Frankreich und den USA: Obwohl das empfundene Risiko (perceived risk) in Frankreich ebenso hoch ist wie in USA, ist das Systemvertrauen (trust) in Frankreich viel größer (Slovic [1997] 2000d, 410); maßgebend dafür dürfte neben der eher technokratischen Men- 
wirten in den sechziger Jahren durchaus beachtet, ist aber seither durch den engeren Begriff der Marktkonformität fast vollständig verdrängt worden. Größere wirtschafts- und sozialpolitische Reformen müssen aber stets in ein glaubwürdiges Gesamtkonzept eingebaut sein, und sie bedürfen begleitender unsicherheitsreduzierender Absicherungsmaßnahmen. Das bedeutet auch, dass sich die volkswirtschaftliche Theorie der Wirtschaftspolitik mit zwei weiteren Konzepten aus der sozialwissenschaftlichen Risikodebatte beschäftigen muss, mit Glaubwürdigkeit und Vertrauen bzw. „,social capital“", Konzepte, die sie bisher - anders als die Sozialwissenschaften ${ }^{42}$ - gleichfalls bloß eher mechanisch behandelt hat.

\section{Literatur}

AEC (US-Atomic Energy Commission and Nuclear Regulatory Commission), 1975, Reactor safety study, (WASH-1400), Washington.

AEC (US-Atomic Energy Commission), 1957, Theoretical possibilities and consequences of major accidents in large nuclear power plants, Brookhaven National Laboratory (WASH 740).

Agell, J., 2003, Efficiency and equality in the labour market, CESifo Forum 4(2), 33-42.

Aiginger, K., 2003a, A three tiers strategy for successful European countries in the nineties, Manuskript.

Aiginger, K., 2003b, The importance of labour market reforms to economic growth, Manuskript.

Bauer, M. W. and G. Gaskell, eds, 2002, Biotechnology. The making of a global controversy, Cambridge, UK: Cambridge University Press.

Bechmann, G. et al, 1986, Gesellschaftlicher Wertewandel am Beispiel der Energiepolitik, in Bechmann und Meyer-Krahmer 1986, 247-92.

Bechmann, G. und F. Meyer-Krahmer, Hg, 1986, Technologiepolitik und Sozialwissenschaft, New York/Frankfurt: Campus.

Bechmann, G., Hg, 1993, Risiko und Gesellschaft. Grundlagen und Ergebnisse interdisziplinärer Risikoforschung, Opladen: Westdeutscher Verlag.

Beck, U., 1986, Risikogesellschaft. Auf dem Weg in eine andere Moderne, Frankfurt: Suhrkamp.

Beck, U., 1988, Gegengifte, Die organisierte Unverantwortlichkeit, Frankfurt: Suhrkamp.

Berger, J., Hg, 1986, Die Moderne-Kontinuitäten und Zäsuren, Soziale Welt Sonderband 4, Göttingen.

Binswanger, H. C. et al, 1978, Der NAWU-Report, Frankfurt: Fischer TBV.

Binswanger, H. C., 1990, Neue Dimensionen des Risikos, Zeitschrift für Umweltpolitik 2, 103-18.

Bjørnskov, Ch., 2002, The happy few: Cross-country evidence on social capital and life satisfaction, Kyklos 56(1), 3-16.

talität der Franzosen der stärkere staatliche Einfluss auf die Technologieentwicklung, aber auch die mangelnde Aufschaukelung infolge staatlicher Aktionen gegen die Proteste gewesen sein (Slovic [1993] 2000c, 324).

42 Siehe etwa Kaase and Newton 1995, Knack and Keefer 1997, Seligman 1997, Braithwaite and Levi 1998, Dasgupta 2000, Putnam 2000, Pollitt 2001, Delhey and Newton 2002. 
Braithwaite, V. and M. Levi, eds, 1998, Trust and governance, New York: Russell Sage.

Camerer, C. F. and M. Kunreuther, 1989, Decision processes for low probability events: Policy implications, Journal of Policy Analysis and Management 8(4), 565-92.

Capra, F., ${ }^{5} 1983$, Wendezeit, Bern et al: Scherz.

Commoner, B., 1973, Wachstumswahn und Umweltkrise, Gütersloh: Bertelsmann (amerik. Original 1971, The common circle).

Daele, W.v.d., 1991, Risiko-Kommunikation: Gentechnologie, in Jungerman et al 1991, 11-61.

Dahrendorf, R., 1992, Der moderne soziale Konflikt, Stuttgart: DVA.

Dasgupta, P. and I. Serageldin, 2000, Social capital: A multifaceted perspective, Washington, DC: World Bank.

Delhey, J. and K. Newton, 2002, Who trusts? The origins of social trust in seven nations, Wissenschaftszentrum Berlin Arbeitspapier FS III 02 - 402.

Dietz, T. and R. Rycroft, 1987, The risk professionals, New York: Russell Sage.

Douglas, M. and A. Wildavsky, 1982, Risk and culture: An essay on the selection of technical and environmental dangers, Berkeley, Cal.: University of California Press.

Enquete-Kommission des Deutschen Bundestages, 1987, Chancen und Risiken der Gentechnologie, München: Schweitzer (Bundestagsdrucksache 10/6775).

European Opinion Research Group, 2001, Eurobarometer 54.2, The social situation in the European Union, (February 2001).

European Opinion Research Group, 2002a, Eurobarometer 56.1, Social precarity and social integration.

European Opinion Research Group, 2002b, Eurobarometer 58.0, Public safety, exposure to drugrelated problems and crime (Autumn 2002)

Evers, A. und H. Nowotny, 1987, Über den Umgang mit Unsicherheit, Frankfurt/M.: Suhrkamp.

Ewald, F., 1993, Der Vorsorgestaat, Frankfurt/M.: Suhrkamp (französisches Original 1986).

Freeman, R.B., 1998, War of models: Which labour market institutions for the $21^{\text {st }}$ century? Labour Economics 5, 1-24.

Gaskell G. et al, forthcoming, GM foods and the misperception of risk perception. J. Risk Research

GfK (Gesellschaft für Sozialforschung), 2002, Ergebnis-Highlights der Lifestyle-Studie 2002, mimeo.

Gibbons, M. et al, 1994, The new production of knowledge, London: Sage.

Gruhl, H., [1975] 1989, Ein Planet wird geplündert, Frankfurt: Fischer TBV.

Habermas, J., H1968, Technik und Wissenschaft als ,Ideologie', Frankfurt: Suhrkamp.

Harris, L., 1980, Risk in a complex society, New York: Marsh and McClennan.

Himmelfarb, G., 1995, The de-moralisation of society: From Victorian virtues to modern values, London: IEA.

Hobsbawn, E., 1995, The age of extremes, London: Joseph.

Huschka, D. 2002, Entwicklungen der deutschen Lebensqualität - die Bundesländer im Vergleich, Wissenschaftszentrum Berlin Arbeitspapier FS III 02 - 404. 
IMAS, 2001, Probleme des technischen Wandels: $54 \%$ klagen: Das Tempo des Fortschritts ist zu hoch, IMAS-Report 1, Jänner.

IMAS, 2002a, Der Sympathiewert von Begriffen: Die Minusworte der Österreicher: Kernenergie, Genforschung, Streiks, IMAS-Report 1, Jänner.

IMAS, 2002b, Kritischer Blick auf die Freizeitgesellschaft: Freizeitbesitz wurde größer, Freizeitnutzung aber passiver, IMAS-Report 19, Oktober.

Inhaber, H. and S. Norman, 1982, The increase in risk interest, Risk Analysis 2, 119-120.

Japp, K.P., 1999, Die Unterscheidung von Nichtwissen, TA-Datenbank-Nachrichten 8(3/4), 25-32.

Jonas, H., 1984, Das Prinzip Verantwortung. Versuch einer Ethik für die technologische Zivilisation, Frankfurt/M.: Suhrkamp.

Jungermann, H. et al, Hg, 1991, Risikokontroversen. Konzepte, Konflikte, Kommunikation, Berlin: Springer.

Jungermann, H., 1991, Inhalte und Konzepte der Risiko-Kommunikation, in Jungermann et al 1991, 335-54.

Kaase, M. and K. Newton, 1995, Beliefs in government, Oxford: Oxford University Press.

Kahneman, D. and A. Tversky, 1972, Subjective probability: A judgement of representativeness, Cognitive Psychology 3, 430-54.

Katzenstein, P., 1985, Small states in world markets: Industrial policy in Europe, Ithaka, NY: Cornell University Press.

Kaufmann, F. X., 1970, Sicherheit als soziologisches und sozialpolitisches Problem, Stuttgart: F. Enke.

Kaufmann, F. X., 2003, Varianten des Wohlfahrtsstaats. Der deutsche Sozialstaat im internationalen Vergleich, Frankfurt: Suhrkamp.

Knack, St. and Ph. Keefer, 1997, Does social capital have an economic payoff? A cross-country investigation, Quarterly Journal of Economics 112(4), 1251-88.

Knight, F. H., 1921, Risk, uncertainty, and profit, Boston/New York: Houghton Mifflin.

Kollert, R., 1993, Systematische Unterbewertung von Katastrophenrisiken - Zur Anwendung des Risikobegriffs in nuklearen Risikoanalysen, in Bechmann Hg 1993, 25-57.

Kunreuther, H. C. and E. V. Levy, 1982, The risk analysis controversy. An institutional perspective, Berlin et al : Springer.

LaPorte, T. R., 1982, On the design and management of a nearly error free organizational control system, in Sills et al 1982, 185-200.

Lau, Ch., 1989, Risikodiskurse: Gesellschaftliche Auseinandersetzung um die Definition von Risiken, Soziale Welt 40, 418-36.

Luhmann, N., [1968] ${ }^{4}$ 2000, Vertrauen. Ein Mechanismus der Reduktion sozialer Komplexität, Stuttgart: Lucius\&Lucius.

Luhmann, N., [1984] 1987, Soziale Systeme, Frankfurt/M: Suhrkamp.

Luhmann, N., [1988] 2¹996, Die Wirtschaft der Gesellschaft, Frankfurt/M: Suhrkamp.

Luhmann, N., 1993, Die Moral des Risikos und das Risiko der Moral, in Bechmann 1993, 327-38.

Meyer-Abich, K. M., 1984, Frieden mit der Natur, München: Hanser. 
Nickell, St., 2003, Labour market institutions and unemployment in OECD countries, CESifo Dice Report 1(2), 13-26.

Nicoletti, G. et al, 2000, Summary indicators of product market regulations with an extension to employment protection legislation, OECD Economics Department Working Paper 226, Paris.

Offe, Cl., 1986, Die Utopie der Null-Option, in Berger 1986, 97-117.

Otway, H. J. and D.v. Winterfeld, 1992, Expert judgement in risk analysis and management: Process, context, and pitfalls, Risk Analysis 12(1), 83-93.

Perrow, Ch., [1987] 1989, Normale Katastrophen. Die unvermeidbaren Risiken der Großtechnik, Frankfurt/M., New York: Campus (amerikanische Originalausgabe 1984).

Perrow, Ch., 1986, Lernen wir aus den jüngsten Katastrophen? Soziale Welt 37, 390-401.

Polanyi, K., [1977] 1978, The Great Transformation. Politische und ökonomische Ursprünge von Gesellschaften und Wirtschaftssystemen, Frankfurt: Suhrkamp (englisches Original 1944).

Pollitt, M., 2001, The economics of trust, norms and networks, mimeo.

Putnam, R. D., 2000, Bowling alone: The collapse and revival of American community, New York: Simon \& Schuster.

Renn, O., 1981, Wahrnehmung und Akzeptanz technischer Risken, Jülich: Kernforschungsanlage.

Rodrik, R. D., 1998, Why do more open economies have bigger governments?, Journal of Political Economy 106(5), 997-1032.

Rucht, D., 1988, Gegenöffentlichkeit und Gegenexperten: Zur Institutionalisierung des Widerspruchs in Politik und Recht, Zeitschrift für Rechtssoziologie 2(9), 44-49.

Schettkatt, R., 2003, Institutions in the economic fitness landscape: What impact do welfare state institutions have on economic performance? IZA Discussion Paper 696.

Schwing, R. C. and W. J. Albers, eds, 1980, Societal risk assessment: How safe is safe enough? New York.

Seifert, F., 2002, Gentechnik-Öffentlichkeit-Demokratie. Der österreichische Gentechnik-Konflikt im internationalen Kontext, Manuskript.

Seligman, A. B., 1997, The problem of trust, Princeton: Princeton University Press.

Sills, D. S. et al, eds, 1982, Accident at Three Mile Island. The human dimension, Boulder, Col.: Westview.

Sinai, I. R., 1990, The human drama, New York: Lang.

Slovic, P., 1987, Perception of risk, Science 236, 280-85 (wieder abgedruckt in Slovic 2000a, 220-31).

Slovic, P., [1993] 2000c, Perceived risk, trust and democracy, in Slovic 2000a, 316-26.

Slovic, P., [1997] 2000d, Trust, emotion, sex, politics and science: Surveying the risk-assessment battlefield, in Slovic 2000a, 390-412.

Slovic, P., 2000b, Introduction and overview, in Slovic 2000a, i-xxxvii.

Slovic, P., ed, 2000a, The perception of risk, London/Sterling VA: Earthscan.

Starr, C., 1980, Introductory remarks, in Schwing and Albers 1980, 2-4.

TAB (Büro für Technikfolgen-Abschätzung beim Deutschen Bundestag), 2002, Technikakzeptanz und Kontroversen über Technik. Umfrage zur Technikakzeptanz, TAB Brief 23, 24-27. 
Thompson, M. and A. Wildavsky, 1982, A proposal to create a cultural theory of risk, in Kunreuther et al 1982, 145-61.

Tichy, G., 2002, Experts' over-optimism in assessment and foresight, TA manu:script, ITA-02-05, Oktober, http://www.oeaw.ac.at/ita/pdf/ita_02_05.pdf; erscheint in Technological Forecasting and Social Change

Tichy, G., 2003a, Wirtschaftsverfassung als Wettbewerbsinstrument - Zum beginnenden Wirtschaftskrieg mit den USA, in Weinzierl, 15-50.

Tichy, G., 2003b, Wandlungen im sozialpolitischen Zielkatalog - Der zunehmende Stellenwert von Lebensqualität und Sicherheit, Manuskript.

Tichy, G., 2004, Der Wandel von der ,Sozialdemokratischen Geborgenheit' zur ,Neuen Unsicherheit' - Eine vernachlässigte Ursache der Stagnation, erscheint in Wirtschaft und Gesellschaft

Toffler, A., [1970] 1979, The future shock, New York: Bantam Books.

Torgersen, H., 2001, Precautionary openness. Understandings of precaution as an indicator for change in biotechnology policy, Politeia 2001/4, 67-79.

Torgersen, H. et al, 2002, Promise, problems and proxies: twenty-five years of debate and regulation in Europe, in Bauer and Gaskell 2002, 21-94.

Torgersen, H., 2002, Austria and the transatlantic agricultural biotechnology divide, Science Communication 24(2), 173-83.

Tversky, A. and D. Kahneman, 1981, The framing of decisions and the psychology of choice, Science 211, 453-58.

Vester, F., 1972, Ausfahrt Zukunft, München: Heyne.

Weinstein, N.D., 1984, Why it won't happen to me: Perceptions of risk factors and susceptibility, Health Psychology 3, 431-57.

Weinzierl, R., Hg, 2003, Hegemonie des American Way of Life oder europäischer Weg? Wien: Löcker.

Wiedemann, P.M., 1993, Tabu, Sünde. Risiko: Veränderungen der gesellschaftlichen Wahrnehmung von Gefährdungen, in Bayerische Rück 1993, 43-67.

Wildavsky, A., 1988, Searching for safety, New Brunswick, London: Transaction Books.

Wynne, B., 1987, Risk management and hazardous waste. Implementation and the dialectics of credibility, Berlin: Springer.

Zapf, W. et al, Hg, 1987, Individualisierung und Sicherheit, München: Beck. 


\section{Bisher erschienene manu:scripte}

ITA-0I-0I Gunther Tichy, Walter Peissl (12/2001): Beeinträchtigung der Privatsphäre in der Informationsgesellschaft. <http://www.oeaw.ac.at/ita/pdf/ita_0I_OI.pdf>

ITA-0I-02 Georg Aichholzer(12/2001): Delphi Austria: An Example of Tailoring Foresight to the Needs of a Small Country. <http://www.oeaw.ac.at/ita/pdf/ita_0I_02.pdf>

ITA-0I-03 Helge Torgersen, Jürgen Hampel (12/2001): The Gate-Resonance Model: The Interface of Policy, Media and the Public in Technology Conflicts.

<http://www.oeaw.ac.at/ita/pdf/ita_0I_03.pdf>

ITA-02-0I Georg Aichholzer (01/2002): Das ExpertInnen-Delphi: Methodische Grundlagen und Anwendungsfeld ,Technology Foresight““.

<http://www.oeaw.ac.at/ita/pdf/ita_02_0l.pdf>

ITA-02-02 Walter Peissl (01/2002): Surveillance and Security - A Dodgy Relationship.

$<$ http://www.oeaw.ac.at/ita/pdf/ita_02_02.pdf>

ITA-02-03 Gunther Tichy (02/2002): Informationsgesellschaft und flexiblere Arbeitsmärkte.

$<$ http://www.oeaw.ac.at/ita/pdf/ita_02_03.pdf $>$

ITA-02-04 Andreas Diekmann (06/2002): Diagnose von Fehlerquellen und methodische Qualität in der sozialwissenschaftlichen Forschung.

<http://www.oeaw.ac.at/ita/pdf/ita_02_04.pdf>

ITA-02-05 Gunther Tichy (10/2002): Over-optimism Among Experts in Assessment and Foresight. $<$ http://www.oeaw.ac.at/ita/pdf/ita_02_05.pdf $>$

ITA-02-06 Hilmar Westholm (12/2002): Mit eDemocracy zu deliberativer Politik? Zur Praxis und Anschlussfähigkeit eines neuen Mediums.

<http://www.oeaw.ac.at/ita/pdf/ita_02_06.pdf>

ITA-03-0I Jörg Flecker und Sabine Kirschenhofer (01/2003): IT verleiht Flügel? Aktuelle Tendenzen der räumlichen Verlagerung von Arbeit. $<$ http://www.oeaw.ac.at/ita/pdf/ita_03_0l.pdf>

ITA-03-02 Gunther Tichy (11/2003): Die Risikogesellschaft - Ein vernachlässigtes Konzept in der europäischen Stagnationsdiskussion.

<http://www.oeaw.ac.at/ita/pdf/ita_03_02.pdf> 